Draft version June 14, 2022. This paper is submitted for publication and has not yet been peer reviewed. We welcome your feedback and thank you for your interest. Please do not copy without author's permission.

\title{
Are Changes in the Perception of Major Life Events Associated With Changes in
}

\section{Subjective Well-Being?}

\author{
Peter Haehner ${ }^{1}$, Lena Sophie Pfeifer ${ }^{1}$, Ina Fassbender ${ }^{2}$, and Maike Luhmann ${ }^{1}$ \\ ${ }^{1}$ Department of Psychology, Ruhr University Bochum \\ ${ }^{2}$ Department of Psychology, University of Siegen
}

\begin{abstract}
After experiencing a major life event, people differ in the extent and duration of event-related changes in subjective well-being (SWB). Some of these individual differences can be explained by individual differences in how people perceive major life events. But how events are perceived does not only vary between individuals, it also changes over time. We therefore examined the longitudinal interplay between perceived event characteristics and SWB using two short-term longitudinal studies conducted with German-speaking participants $\left(N_{\text {Study } 1}=619,73 \%\right.$ female; $N_{\text {Study } 2}=691,71 \%$ female $)$. In both studies, perceived event characteristics and SWB were assessed at two measurement occasions 3 months apart. We used bivariate latent change score models to estimate correlated changes of these constructs. Perceiving events as increasingly externally controlled and as increasingly social status threatening over time was consistently associated with a decrease in life satisfaction. Furthermore, perceiving events as increasingly challenging over time was consistently associated with a decrease in positive affect and an increase in negative affect. Our results indicate that the development of SWB and perceived event characteristics is intertwined, and they highlight the importance of assessing perceived event characteristics longitudinally to gain a better understanding of event-related changes in SWB.
\end{abstract}

Keywords: major life events, perceived event characteristics, subjective well-being, correlated changes

\section{Author Note.}

Peter Haehner (iD https://orcid.org/0000-0002-3896-6172

Lena Sophie Pfeifer (iD https://orcid.org/0000-0001-8803-6515

Ina Fassbender (iD https://orcid.org/0000-0003-0423-6259

Maike Luhmann (iD https://orcid.org/0000-0001-6211-9304

The study design of Study 1 was preregistered at https://osf.io/sg3nk. The study design of Study 2 was preregistered at https://osf.io/x52bq. The preregistration of the analyses, the data, and $\mathrm{R}$ scripts can be retrieved from https://osf.io/cp7d6. We have no known conflict of interest to disclose. Data collection was funded by Pathways to Character Project (sponsored by the John Templeton Foundation and the Arizona State University Foundation), Grant 60699 to Maike Luhmann. The contribution of Lena Sophie Pfeifer was supported by the DFG within project B4 of the Collaborative Research Center (SFB) 874 "Integration and Representation of Sensory Processes" [project number 122679504]. We would like to thank Lennart Martens for his support with the literature search and data preparation. Correspondence concerning this article should be addressed to Peter Haehner, Department of Psychology, Ruhr University Bochum, Universitaetsstr. 150, 44780 Bochum, Germany. Email: peter.haehner@rub.de 


\section{Are Changes in the Perception of Major Life Events Associated With Changes in Subjective Well-Being?}

Major life events are "events that are clearly timed, disrupt one's everyday routine, and are perceived as personally significant and memorable by those who experienced them" (Luhmann et al., 2021, p. 634). Major life events such as a job loss can lead to lasting changes in subjective well-being (SWB; Lucas, 2007; Specht et al., 2011). However, not all people react in the same way to a major life event (Luhmann et al., 2021; Yap et al., 2014).

Previous studies suggested that some of these individual differences in event-related changes in SWB can be explained by differences in how people perceive a major life event, for example, the extent to which an event was perceived as negative, impactful, or controllable (Luhmann et al., 2021; Prizmić-Larsen et al., 2020). However, in these studies, perceived characteristics of major life events were assessed only once, implying that the perception of $n$ event is stable over time. But recent research has shown that this assumption is probably not tenable: the perception of major life events changes over time (Haehner, Kritzler et al., 2021). This novel observation raises the question of whether changes in the perception of major life events are related to changes in SWB. For example, how does SWB change if a negatively perceived event is perceived as increasingly positive (or increasingly negative) over time?

In this article, we addressed this question using two short-term longitudinal studies in which we assessed changes in SWB and changes in the perception of major life events over a period of 3 months. As we elaborate in more detail below, examining the dynamic interplay of these constructs is relevant for both, theoretical and empirical reasons. First, this type of association is proposed in several theories but rarely tested directly (e.g., Lazarus \& Folkman, 1984; Wilson \& Gilbert, 2008). Second, the results may shed light on the nature of the relationship between perceived event characteristics and SWB: correlated changes between perceived event characteristics and SWB might be an indication of feedback loops between these constructs (see Olaru \& Allemand, 2021). Finally, correlated changes between these constructs would have implications for the measurement of major life events (e.g., assessing perceived event characteristics at multiple measurement occasions).

\section{Major Life Events and Changes in SWB}

SWB describes how people experience and evaluate their lives (Diener, 1984). SWB is often conceptualized as comprising three related components: life satisfaction, positive affect, and negative affect. Life satisfaction describes the cognitive evaluation of life. Positive and negative affect (also referred to as affective well-being) encompass positive and negative feelings and mood (Busseri, 2018; Diener et al., 1999). As these components of SWB differ in their correlates and their stability over time, they should be analyzed separately when investigating the effects of major life events (e.g., Kettlewell et al., 2020; Luhmann et al., 2012). 
Longitudinal studies indicate that major life events can lead to lasting changes in SWB-at least for some people (Denissen et al., 2019; Kettlewell et al., 2020; Luhmann et al., 2012). However, important questions remain: For example, why do these effects differ among events and among people (Luhmann et al., 2021; Yap et al., 2014)? One way to approach these questions is to change the assessment of major life events. Most longitudinal studies simply examined whether or not a major life event occurred (e.g., by inferring the occurrence of life events based on changes in demographical variables; Denissen et al., 2019; Kettlewell et al., 2020). This rather simple assessment of major life events neglects that the supposedly same major life event may be perceived differently by different individuals and thus have different implications for people's SWB (Luhmann et al., 2021). For example, for one person, a job loss may be a very negative and impactful experience, whereas another person may perceive this event as rather neutral.

\section{Perceived Event Characteristics and Changes in SWB}

To assess how people perceive major life events, we focus on perceived event characteristics. Perceived event characteristics are ratings of people's subjective experience of an event on different continuous dimensions. For example, the Event Characteristics Questionnaire (Luhmann et al., 2021) assesses nine perceived event characteristics: challenge, change in world views, external control, extraordinariness, emotional significance, predictability, impact, social status change, and valence.

Several theories suggest that changes in SWB may be associated with perceived event characteristics (for an overview, see Luhmann \& Intelisano, 2018). For example, the concept of hedonic adaptation (Frederick \& Loewenstein, 1999) implies a direct relationship between the perceived valence of an event and the hedonic response (i.e., changes in SWB). Furthermore, affective adaptation theory (Wilson \& Gilbert, 2008) assumes that the emotional response to an event depends on the extent to which the event is perceived as unexplained, unexpected, and self-relevant. Relatedly, several depression theories predict that the onset of depression after negative life events depends on their perceived controllability (Abramson et al., 1978), their self-relevance (Beck \& Bredemeier, 2016), their implications on the social status (Slavich \& Sacher, 2019), and their perceived consequences (Abramson et al., 1989).

Empirically, there is robust evidence that individual differences in the perception of major life events can explain the onset of depression (for a review, see Haehner et al., 2022). For SWB, the perceived importance of negative events longitudinally predicted higher levels of negative affect (Prizmić-Larsen et al., 2020). Similarly, perceiving negative events as more impactful was associated with a more pronounced adaptation in people's affective well-being after the event (Luhmann et al., 2021). In addition, the perceived event characteristics challenge, extraordinariness, and social status changes were also associated with changes in SWB in this study. 


\section{Changes in the Perceived Event Characteristics and Changes in SWB}

The perception of major life events differs not only among people, it also changes over time (Haehner, Kritzler et al., 2021), which leads to the question: Are changes in the perception of major life events related to changes in SWB? Examining the dynamic interplay of the perception of major life events and SWB is theoretically relevant as several theories assume the existence of feedback loops and coupled changes among these constructs. First, the concept of reappraisal as introduced in the appraisal theory (Lazarus \& Folkman, 1984) or in the emotion regulation literature (e.g., Gross, 2002) implies that changes in the perception of major life events (i.e., a reappraisal) lead to changes in SWB. Second, affective adaptation theory states that affective adaptation to an event "involves higher order mental processes that alter the meaning of those events" (Wilson \& Gilbert, 2008, p. 370). In particular, perceiving events as more explainable, as less extraordinary, or as less self-relevant should be related to changes in SWB. While these theories suggest that changes in the perception of major life events lead to changes in SWB, there are also theoretical propositions that support the opposite direction of causality. For example, mood-congruent memory effect suggests that changes in people's SWB influences how they remember past experiences (Mayer et al., 1995). Thus, if people's SWB increases, this might cause a more positive memory and evaluation of past events.

Empirically, we know only little about possible associations between changes in the perception of major life events and changes in SWB. For the related domain of mental health, Brose et al. (2021) examined associations between the perception of the Covid-19 pandemic and changes in mental health. They found that not the perception of the Covid-19 pandemic as such but changes in this perception (e.g., perceiving the pandemic as increasingly threatening and challenging over time) predicted decreases in mental health. Similarly, in a study by Shigemoto (2020), perceiving more event-related growth over time was associated with less post-traumatic stress symptoms. However, for SWB, empirical evidence on correlated changes between perceived event characteristics and SWB is currently lacking.

\section{The Present Article}

In this article, we examined whether changes in perceived event characteristics are associated with changes in SWB. We conducted two short-term longitudinal studies in which we assessed participants' SWB and their perception of a recently experienced major life event at two measurement occasions 3 months apart. In Study 1, we explored the associations between changes in the perceived event characteristics and changes in SWB. Based on these results, we preregistered our analyses and hypotheses for Study 2 and evaluated whether the associations found in Study 1 could be replicated in this second study. 


\section{Study 1: Exploratory Analyses}

Study 1 was based on data from the What's NEXT? Study, a longitudinal online study with five measurement occasions spread over 1 year. Data from the What's NEXT? Study have already been used in previous publications on perceived event characteristics (Fassbender et al., 2021; Haehner, Kritzler et al., 2021; Haehner, Rakhshani et al., 2021; Kritzler et al., 2021; Luhmann et al., 2021). However, none of these studies examined the relationship between changes in the perception of major life events and changes in SWB (see Table S1 for details).

\section{Methods}

\section{Procedure}

The What's NEXT? Study was conducted in 2018 and 2019. The study was addressed to young adults which first had to register for it. Registration comprised age verification (minimum age 18 years), providing informed consent, and providing an email address. Afterwards, participants were invited via email to the five measurement occasions of the study (i.e., 0, 12, 24, 36, 48 weeks after registration).

For the present article, we only used data from the first and second measurement occasion (T1 and T2) of the What's NEXT? Study because participants rated their perception of the same major life event at these measurement occasions only. At T1, participants freely named the most important major life event they had experienced in the last 3 months and rated their perception of this event with the Event Characteristics Questionnaire. At T2, participants were shown the event they had named at T1 and rated this event again. SWB was assessed at both measurement occasions (see the study-design preregistration below for a list of all measures).

\section{Participants}

German-speaking young adults were recruited via social media and at introductory events at universities. In total, 857 people registered to take part in the study. To ensure data quality, we excluded participants with missing or incorrect answers on two instructed response items (e.g., "In order to secure the data quality, please select the option often") and participants who completed a measurement occasion in less than 10 minutes (i.e., $40 \%$ of the expected duration). Furthermore, we excluded participants who did not participate at T1 or who named a major life event at $\mathrm{T} 1$ that occurred more than 3 months ago (i.e., outside the requested time frame). Applying these criteria, our final sample size was $N=619$ at $\mathrm{T} 1$ and $N=433$ at T2. Participants' mean age at T1 was 21.48 years $(S D=4.05)$ and $73 \%$ of the sample was female. About $93 \%$ of the sample had a high school graduation and around $10 \%$ of the sample indicated that their mother tongue was not German (i.e., an indirect measure of migration background). 


\section{Measures}

For all measures, we reversed responses if appropriate and calculated mean scores for descriptive purposes.

Naming a Major Life Event and Timing of the Event (T1). Participants freely named a major life event they had experienced in the previous 3 months. We coded these free-text answers into event categories (see Table S2 for details). Furthermore, participants were asked to indicate how many weeks ago the event occurred (variable weeks.ago).

Event Characteristics (T1 and T2). Participants rated their perception of the named event using the Event Characteristics Questionnaire (Luhmann et al., 2021; 37-item version as in Study 5). This questionnaire assesses nine perceived event characteristics: valence (6 items, e.g., "The event was joyful"), impact (4 items, e.g., "The event had long-term consequences"), challenge (4 items, e.g., "The event was straining"), change in world views (4 items, e.g., "The event helped me to gain new perspectives"), emotional significance (4 items, e.g., "The event moved me a lot"), external control (4 items, e.g., "Others were able to control the event"), extraordinariness (3 items, e.g., "The event was extraordinary"), predictability (4 items, e.g., "The event was surprising"), and social status change (4 items, e.g., The event threatened my social status). Items were rated on a scale ranging from 1 (not true at all) to 5 (absolutely true).

Positive and Negative Affect (T1 and T2). Positive and negative affect were assessed with three items, respectively, using the German 6-item version of the Scale of Positive and Negative Experiences (Diener et al., 2010; German version by Rahm et al., 2017). Participants rated how often they had felt a certain way in the last month (e.g., "sad") on a scale ranging from 1 (very rarely or never) to 5 (very often or always).

Life Satisfaction (T1 and T2). Life satisfaction was assessed with the German 3-item version of the Satisfaction with Life Scale (Diener et al., 1985; Oishi, 2006; German version by Glaesmer et al., 2011). Items (e.g., "I am satisfied with my life") were rated on a scale ranging from 1 (strongly disagree) to 7 (strongly agree).

\section{Transparency and Openness}

Data collection of the What's NEXT? Study was approved by local ethics committee of [BLINDED FOR PEER REVIEW]. The study design was preregistered at [LINK BLINDED FOR REVIEW; BUT SEE SUPPLEMENTAL MATERIAL FOR AN ANONYMOUS PDF]. All data, analysis code, and a codebook are available at https://osf.io/e23bc/?view_only=2942696d914643458a4b28dfe80ea633. Analyses for this article were conducted in $\mathrm{R}$ (Version 4.1.1). As Study 1 was exploratory, the analyses were not preregistered. 


\section{Data Analysis}

Our analyses comprised four steps: checking for longitudinal measurement invariance; attrition analysis, estimating univariate latent change score models, and estimating bivariate latent change score models.

Longitudinal measurement invariance. Using the R packages lavaan (Rosseel, 2012) and semTools (Jorgensen et al., 2020), we checked for longitudinal measurement invariance of our measures (see Table S3 for details). All constructs showed scalar measurement invariance, except for life satisfaction and predictability. For life satisfaction, restricting the intercepts to be equal across the two measurement occasions significantly reduced model fit. However, by freeing the intercept of the item "I am satisfied with my life", we were able to establish partial scalar invariance. All subsequent analyses for life satisfaction were thus based on this partially invariant measurement model. For predictability, the fit of the configural model was not acceptable. We decided to drop the item "I knew in advance that the event would be happening" from the analyses of Study 1 because it was involved in the largest modification indices ${ }^{1}$.

Attrition analysis. Using two-sample $t$-tests and $x^{2}$-tests, we examined whether participants who completed both measurement occasions and participants who dropped out of the study differed in their demographic characteristics, their SWB, or the perceived event characteristics. No statistically significant differences were found (Table S4).

Univariate Latent Change Score Models. Latent change score models allow estimating within-person changes without accumulating measurement errors in the calculation of difference score (McArdle, 2009). We computed univariate latent change score models for each construct to evaluate whether they acceptably fitted the data (acceptable: RMSEA <.08, CFI > .95, TLI > .95; Schermelleh-Engel et al., 2003).

With two measurement occasions, univariate latent change score models comprise one latent factor for each measurement occasion (e.g., positive affect T1 and positive affect T2) as well as a latent change score that estimates within-person changes between T1 and T2. Mean and variance of the T1 factor and the latent change score factor were freely estimated; mean and variance of the T2 factor were fixed to 0 . Additionally, to control for possible effects of unequal temporal distance between event occurrence and $\mathrm{T} 1$, we included the temporal distance between the event occurrence and T1 (variable weeks.ago) as predictor of the latent change score factor. We estimated the latent changes score models using the indicator variable method (i.e., fixing the factor loading of the first indicator to 1 and its mean to 0 ). Means and intercepts for the other indicators were freely estimated but constrained to be equal

\footnotetext{
${ }^{1}$ As robustness check, we estimated the latent change score models with all four items of predictability. The pattern of significant findings obtained with the bivariate latent change score models remained unchanged.
} 
across the two measurement occasions (except for life satisfaction as described above). We allowed correlated residuals for the same repeatedly measured indicators. To deal with missing values, we used full information maximum likelihood estimation. An example for a univariate latent change score model is depicted in Figure $1 \mathrm{~A}$.

Bivariate Latent Change Score Models. To examine whether changes in the perceived event characteristics are associated with changes in SWB, we computed 27 bivariate latent change score models-each comprising one SWB component and one perceived event characteristic. Bivariate latent change score models allow estimating correlated changes among constructs independent of measurement error (McArdle, 2009).

In general, these bivariate latent change score models were estimated in the same way as their univariate counterparts. However, we additionally included two phantom variables (see Figure 1B). These phantom variables were based on the latent change score factors but had a variance fixed to 1 . Thus, they could be used to estimate the correlation (instead of the covariance) of the latent change score factors (Little, 2013). To answer our research question, we estimated the statistical significance of this correlation $\left(r_{\Delta \Delta}\right)$. To reduce Type I error inflation, we used a level of significance of $\alpha=.01$ in Study 1 .

\section{Results}

\section{Descriptive Statistics and Univariate Latent Change Score Models}

Participants most frequently reported the events vacation $(n=52)$, beginning college $253(n=48)$, relocation $(n=47)$, and graduation from high school ( $n=46$; Table S2). Table 1 254 includes descriptive statistics of the study variables (see Table S5 for correlations) and descriptive statistics of the latent change score variables estimated by the univariate latent change score models. As indicated by the standardized means of the latent change score variables, changes over time were most pronounced for life satisfaction (increased), change in world views (increased), impact (increased), and emotional significance (decreased). The univariate latent change score models showed acceptable model fit for all constructs (all $\mathrm{CFI} \geq .98, \mathrm{TLI} \geq .97$, and RMSEA $\leq .05$; Table S6). The variable weeks.ago was only significantly associated with the latent change scores for the perceived event characteristic challenge $(b=-0.03, S E=0.01, z=-2.98, p=.003)$.

\section{Bivariate Latent Change Score Models}

All bivariate latent change score models showed at least acceptable fit (all CFI $\geq .95$, $T L I \geq .95$, and RMSEA $\leq .05$; Table S7-S9). The correlations among the latent change score factors, which are decisive for our research question, are summarized in Table 2.

Life Satisfaction. Perceiving events as less externally controlled $\left(r_{\Delta \Delta}=-.25, p=.001\right)$, as less social status threatening $\left(r_{\Delta \Delta}=-.28, p<.001\right)$, and as more positive $\left(r_{\Delta \Delta}=.34\right.$, $269 p<.001)$ over time was related to an increase in life satisfaction. Changes in the other perceived event characteristic were not related to changes in life satisfaction. 
Positive Affect. Perceiving events as less challenging $\left(r_{\Delta \Delta}=-.26, p<.001\right)$, as less externally controlled $\left(r_{\Delta \Delta}=-.19, p=.009\right)$, and as more positive $\left(r_{\Delta \Delta}=.19, p=.003\right)$ over time

273 was related to an increase in positive affect. Changes in the other perceived event 274 characteristic were not related to changes in positive affect.

275 Negative Affect. Perceiving events as more challenging $\left(r_{\Delta \Delta}=.31, p<.001\right)$ and as less positive $\left(r_{\Delta \Delta}=-.23, p<.001\right)$ over time was related to an increase in negative affect. Changes in the other perceived event characteristic were not related to changes in negative 278 affect.

\section{Summary and Discussion}

280 We found several significant associations between changes in the perceived event 281 characteristics and changes in SWB. First, changes in perceived event valence were 282 consistently associated with changes in the three SWB components. This result is in line with 283 previous findings that valence is an important event characteristic for predicting changes in 284 SWB (e.g., Luhmann et al., 2021) and other constructs like personality traits (e.g., Vries et al., 285 2021).

Second, perceiving events as increasingly externally controlled over time was related to decreasing life satisfaction and positive affect. Furthermore, perceiving events as increasingly social status threatening was related to decreasing life satisfaction. These findings are in line with helplessness theory of depression and with social signal transduction theory (Abramson et al., 1978; Slavich \& Sacher, 2019). We elaborate more on these findings in the General Discussion.

Third, perceiving events as increasingly challenging over time was related to decreasing positive affect and increasing negative affect. As the perceived event characteristic challenge was measured with items that focus on people's feelings (e.g., "The event scared me"), it seems reasonable that changes in this perceived event characteristic were related to changes in the affective components of SWB (instead of life satisfaction as cognitive component). In general, our findings underline the importance of analyzing the three SWB components separately to gain a better understanding of event-related changes in SWB (Kettlewell et al., 2020; Luhmann et al., 2012).

The effect sizes for the statistically significant correlated changes between the perceived event characteristics and SWB were at least of medium size (.19 $\leq r_{\Delta \Delta} \leq .34$; Funder \& Ozer, 2019). Compared to results on correlated changes from other domains, the associations were about as strong as correlated changes among certain personality trait domains (Allemand \& Martin, 2016; Klimstra et al., 2013) as well as correlated changes between positive affect and symptoms of anxiety (Hou et al., 2015). Taken together, Study 1 suggests that there are significant correlated changes between perceived event characteristics 
and SWB. However, as Study 1 was exploratory, we tried to replicate the results in a second, independent sample.

\section{Study 2: Confirmatory Analyses}

The aim of Study 2 was to test the associations between changes in perceived event characteristics and changes in SWB in a second sample. We hypothesized that correlations between changes in the perceived event characteristics and changes in SWB that were statistically significant in Study 1 would replicate in Study 2. As Study 2 was based on an existing dataset that was not specifically collected for the purpose of this study, the design of Study 2 differed in some respects from the one of Study 1 . These differences are summarized in Table 3.

Study 2 is based on data from the study One Year of Corona Pandemic. This study was conducted in 2021 and comprised three measurement occasions (T1 to T3). We only used data from $\mathrm{T} 1$ and $\mathrm{T} 3$ because participants only rated their perception of a recently experienced event at these two measurement occasions. T1 and T3 were 3 months apart (i.e., same temporal distance as in Study 1).

\section{Methods}

\section{Procedure}

To take part in the study One Year of Corona Pandemic, participants first had to register for it by providing informed consent, verifying their age (minimum age 18 years), and providing an email address. Thereafter, participants were invited via email to the three measurement occasions of the study (i.e., 0, 1, 12 weeks after registration).

At T1, participants received a checklist of life events and indicated which of these events they had experienced in the past year. For all experienced events, participants reported how many months ago the event occurred and indicated the event that was most significant to them. This most significant event was then rated using the Event Characteristics Questionnaire. At T3, participants were shown the event they had rated at T1 and were asked to re-rate this event. SWB was assessed at both measurement occasions (see the studydesign preregistration below for a list of all measures).

\section{Participants}

German-speaking participants of any age were recruited for Study 2. Based on the results of Study 1 , we conducted a power analysis using the shiny app pwrSEM (Wang \& Rhemtulla, 2021). Assuming a level of significance of $\alpha=.05$, we were able to detect correlated changes of $r_{\Delta \Delta}=.20$ with 600 individuals and a power of $84 \%$. In total, 1075 people registered to take part in the study. We applied the same exclusion criteria as in Study 1: We excluded participants with no or incorrect answers on the instructed response items and participants who completed the online survey for a measurement occasion in less $40 \%$ of the expected duration. Additionally, we excluded participants who had not experienced a major life event in 
344 the last 12 months or who had indicated that the most significant experienced event occurred 345 more than 12 months ago (i.e., event outside the requested time frame). Applying these 346 criteria, our final sample size was $N=691$ at T1 and $N=438$ at T2. Participants' mean age at 347 T1 was 34.18 years $(S D=12.02)$ and $71 \%$ of the sample were female. About $70 \%$ of the 348 sample had a high school graduation and around $6 \%$ of the sample indicated that they were 349 not born in Germany. Measures

For all measures, we reversed responses if appropriate and calculated mean scores for descriptive purposes.

Life Event Checklist and Timing of the Event (T1). Participants received a life event checklist and marked all the events they had experienced in the past year (see Table S11 for similarities and differences between our life event assessment in Study 1 and Study 2). This life event checklist comprised 32 events which had been taken from common life event inventories (Holmes \& Rahe, 1967; Sarason et al., 1978). In addition, participants had the opportunity to name an additional major life event that was not mentioned. Participants then indicated which of the experienced events was most significant to them (i.e., the event that was subsequently rated with the Event Characteristics Questionnaire). Furthermore, participants provided information on how many months ago the event occurred (variable months.ago). For the analyses, we transformed this variable months.ago into a variable weeks.ago by multiplying it with 4.34 so that its interpretation matches the one of the temporal distance variable in Study 1.

Event Characteristics (T1 and T3). Perceived event characteristics were assessed with the Event Characteristics Questionnaire (Luhmann et al., 2021). The items differed slightly between Study 1 and Study 2 (see Table S10 for details). Items were rated on a scale ranging from 1 (not true at all) to 5 (absolutely true).

Life Satisfaction (T1 and T3). Life satisfaction was assessed with the German 5-item version of the Satisfaction with Life Scale (Diener et al., 1985; German version by Glaesmer et al., 2011). Items were rated on a scale ranging from 1 (strongly disagree) to 7 (strongly 372 agree).

Positive and Negative Affect (T1 and T3). Positive and negative affect were assessed with the German 12-item version of the Scale of Positive and Negative Experiences (Diener et al., 2010; German version by Rahm et al., 2017). Items were rated on a scale ranging from 1 (very rarely or never) to 5 (very often or always).

\section{Transparency and Openness}

Data collection of the study One Year of Corona Pandemic was approved by local ethics committee of [BLINDED FOR PEER REVIEW]. The study design was preregistered at 
analyses

for

Study 2

were

preregistered

at

382 https://osf.io/3yw2a/?view only=e884388c4987410b8cb5aa51951f1d6f. Deviations from this

383 preregistration are summarized in Table S13. All data, analysis code, and a codebook are

384 available at https://osf.io/b392y/?view only=9702db8a852344d3972d7d2ebe8faa51.

\section{Data Analysis}

The analyses comprised the same four-step procedure as in Study 1. However, since the employed items slightly differed between the two studies, there were also a few differences in the analyses, which we describe in the following sections.

Longitudinal measurement invariance. We evaluated longitudinal measurement invariance independently from the results from Study 1 (Table S14). All constructs showed scalar measurement invariance, except for emotional significance and valence. For these two perceived event characteristics, we were able to establish partial scalar invariance by allowing one intercept for each construct to differ between the two measurement occasions. All subsequent analyses of these two variables were based on these partially invariant measurement models.

Attrition analysis. As in Study 1, participants who completed both measurement occasions and participants who completed only $\mathrm{T} 1$ did not differ significantly in their demographic characteristics, their SWB, or the perceived event characteristics (Table S15).

Univariate Latent Change Score Models. As in Study 1, we computed univariate latent change score models to test whether their model fit was acceptable (acceptable: RMSEA < .08, CFI > .95, TLI> .95; Schermelleh-Engel et al., 2003). Due to differences in longitudinal measurement invariance and differences in the employed items, the measurement models of the univariate latent change score models slightly differed between the two studies. All other aspects of the latent change score models were retained as in Study 1.

Bivariate Latent Change Score Models. We used bivariate latent change score models to test our hypotheses regarding the associations between changes in perceived event characteristics and changes in SWB. Again, the correlation between the latent change score variables $\left(r_{\Delta \Delta}\right)$ was crucial for these hypotheses. For those correlations that we expected to be significant based on the results of Study 1, we preregistered using a level of significance of $\alpha=.05$. For those correlations that were not significant in Study, we used a more conservative level of significance of $\alpha=.01$ to reduce the risk of false positive findings.

\section{Descriptive Statistics and Univariate Latent Change Score Models}

The most frequently reported major life events were changes in the work situation $415(n=61)$, death of a loved one $(n=57)$, start of a new job $(n=55)$, relocation $(n=49)$, and own 416 serious illness or injury ( $n=43$; Table S12). Table 4 summarizes descriptive statistics of the 
change score variables estimated with the univariate latent change score models. As indicated by the standardized means of the latent change score variables, the most pronounced changes between T1 and T3 were found for negative affect, emotional significance, and impact (all decreased across the 3 months). The variable weeks.ago was not associated with the latent change scores in any of the models. The univariate latent change score models showed acceptable model fit for all constructs (all CFI $\geq .99$, TLI $\geq .98$, and RMSEA $\leq .05$; Table S17).

\section{Bivariate Latent Change Score Models}

All bivariate latent change score models showed at least acceptable fit (all CFI $\geq .96$, $T L I \geq .96$, and RMSEA $\leq .06$; Table S18-S20). Table 5 summarizes the results on the correlations of the latent change scores.

Life Satisfaction. As expected, based on our results from Study 1, perceiving events as less externally controlled $\left(r_{\Delta \Delta}=-.18, p=.008\right)$ and as less social status threatening $\left(r_{\Delta \Delta}=-.17, p=.014\right)$ over time was related to an increase in life satisfaction. However, contrary to our hypotheses, changes in valence were not significantly related to changes in life satisfaction $\left(r_{\Delta \Delta}=.04, p=.592\right)$. Changes in the other perceived event characteristics were also not significantly related to changes in life satisfaction.

Positive Affect. As expected, we found a significant correlation between changes in positive affect and changes in challenge: Perceiving events as less challenging over time was related to an increase in positive affect $\left(r_{\Delta \Delta}=-.19, p=.003\right)$. However, contrary to your hypotheses, changes in external control $\left(r_{\Delta \Delta}=-.02, p=.697\right)$ and changes in valence $\left(r_{\Delta \Delta}=.10, p=.074\right)$ were not significantly related to changes in positive affect in this study. Changes in the other perceived event characteristics were also not significantly related to changes in positive affect.

Negative Affect. As hypothesized, perceiving events as more challenging over time was significantly related to an increase in negative affect $\left(r_{\Delta \Delta}=.19, p=.006\right)$. Furthermore, there was a significant correlation for social status change that was not found in Study 1: Perceiving events as more social status threatening over time was related to an increase in negative affect $\left(r_{\Delta \Delta}=.17, p=.009\right)$. However, the association between changes in valence and changes in negative affect did not replicate in Study $2\left(r_{\Delta \Delta}=-.06 p=.301\right)$. Finally, changes in the other perceived event characteristics were not significantly related to changes in negative affect.

\section{Robustness Checks}

As outlined in Table 3, Study 1 and Study 2 differed in several aspects. To test the robustness of our findings and to evaluate possible reasons for diverging results, we conducted five robustness checks. Each robustness check addressed one aspect that differed between the two studies: differences in the employed items, potential relevance of stress due to the Covid-19 pandemic, differences in age composition of the samples, differences in the 
examined event categories, and different time frames of the included events (for details on the methods and results, see Table 6). In general, the pattern of significant associations was highly consistent across the different robustness checks, with three exceptions. First, using only those items that had been assessed in both studies led to larger standard errors and somewhat lower effect sizes for the correlated changes, possibly due to reduced reliability of the shorter scales. Second, restricting the analyses of Study 2 to those event categories that overlapped between the two studies led to higher effect sizes for the correlated changes and made the results of Study 2 more similar to the ones of Study 1. Third, restricting the analyses of Study 2 to events that occurred in the last 3 months (i.e., the time frame of in Study 1) also led to stronger correlated changes between the perceived event characteristics and changes in SWB (in particular, for positive and negative affect).

\section{Exploratory Analyses (not Preregistered)}

To gain first insights into the direction of causality between changes in perceived event characteristics and changes in SWB, we estimated bivariate latent change score models that additionally included time-ordered paths. Building on the bivariate latent change score model depicted in Figure 1B, we additionally included a path from the T1 score of SWB to the latent change score of the perceived event characteristics (Path $A$ ) as well as a path from the T1 score of the perceived event characteristics to the latent change score of SWB (Path B). The results of these exploratory analyses can be found in the supplemental material (Table S26 and S27). In summary, neither Path $A$ nor Path $B$ were significant in most bivariate latent change score models (i.e., 102 out of 108 associations were not significant using a level of significance of $\alpha=.01$ ). Thus, we refrain from drawing any conclusions about the direction of causality for the observed correlated changes based on our exploratory analyses.

\section{Summary and Discussion}

In Study 2, we examined whether the correlations between changes in perceived event characteristics and changes in SWB found in Study 1 replicated in another sample under slightly modified conditions. Perceiving events as increasingly externally controlled and as increasingly social status threatening over time was related to a decrease in life satisfaction in both studies. Furthermore, perceiving events as increasingly challenging over time was related to a decrease in positive affect and to an increase in negative affect in both studies. With some minor deviations, this pattern of results was also found in the robustness checks.

Surprisingly, changes in perceived valence, which were consistently related to changes in SWB in Study 1, were not related to changes in any SWB component in Study 2. As indicated by the robustness checks, these differences can at least partly be explained by differences in the employed time frame of the considered events (i.e., 3 months in Study 1 versus 12 months in Study 2) as well as the sampled event categories. 


\section{General Discussion}

In this study, we examined whether changes in perceived event characteristics were related to changes in SWB. Drawing on two short-term longitudinal studies, the following associations consistently emerged: Perceiving events as increasingly challenging over time was related to a decrease in positive affect and an increase in negative affect. Furthermore, perceiving events as increasingly externally controlled and as increasingly social status threatening over time was related to a decrease in life satisfaction. In addition, there were correlations that only emerged in one of the two studies and that might require future research. In particular, the fact that changes in valence were consistently related to changes in all three components of SWB in Study 1 but unrelated to changes in SWB in Study 2 was surprising.

\section{Changes in the Perceived Event Characteristics and Changes in SWB}

The results of our study are consistent with theories that propose the existence of correlated changes between the perception of major life events and changes in SWB (Lazarus \& Folkman, 1984; Wilson \& Gilbert, 2008). In particular, the negative association between changes in perceived challenge and changes in affective well-being are in line with appraisal theory as the challenge appraisal is assumed to be of central relevance for the resulting affective reaction to a stressor (Lazarus \& Folkman, 1984). Furthermore, this finding that perceiving events as increasingly challenging over time was associated with a decrease in affective well-being is also in line with previous findings. Brose et al. (2021) found that perceiving the Covid-19 pandemic as increasingly threatening and challenging over time predicted decreases in mental health.

The associations between changes in life satisfaction and changes in external control and social status change can best be explained by theories on the onset of depression. According to helplessness theory (Abramson et al., 1978) and social signal transduction theory (Slavich \& Sacher, 2019), perceiving events as externally controlled and as social status threatening should contribute to the onset of depression. Thus, it seems reasonable that perceiving events as more externally controlled and as more social status threatening over time was associated with a decrease in life satisfaction.

Differences in the item content of the perceived event characteristics might explain why their correlated changes differed among the three SWB components. For example, items for external control require a cognitive evaluation of the causes of the event (e.g., "Others were responsible for the event") explaining why changes in external control were most strongly related to changes in life satisfaction as cognitive component of SWB. In contrast, items for challenge refer to the feelings that were elicited by the event (e.g., "The event scared me") explaining why changes in this characteristic were most strongly related to changes in positive and negative affect. 
Regarding the perceived event characteristic valence, our two studies led to inconsistent results. In Study 1, valence was consistently associated with changes in all three components of SWB and the effect sizes were quite large. In Study 2, no statistically significant correlated changes between valence and SWB were found. Our robustness checks examined whether these different results can be explained by differences in the design of the two studies. In particular, the temporal distance between the event occurrence and T1 and the sampled event categories seemed to be at least partly responsible for the diverging findings indicating that changes in perceived valence are only correlated with changes in SWB under specific conditions.

\section{Possible Causes for Correlated Changes}

As we found correlated changes between the perception of major life events and SWB, we must address the question why these associations emerged. Our study was based on theories assuming feedback loops and coupled changes among these constructs (e.g., Lazarus \& Folkman, 1984; Wilson \& Gilbert, 2008). Additional other possible causes for correlated changes discussed in the literature are response biases and external factors inducing similar changes in both constructs (Olaru \& Allemand, 2021). For example, public events (e.g., terrorist attacks) or seasonal changes might induce changes in people's SWB and the perceived event characteristics and thus be responsible for the observed correlated changes. Nonetheless, we argue that feedback loops and coupled changes between the perception of major life events and SWB are the most likely explanations for our results. First, there was no indication that the number of reversed-keyed items used in the assessment of the perceived event characteristics (varying from zero to three per subscale) was associated with strength of the associations which speaks against a pronounced influence of response biases such as acquiescence. Second, by examining the associations in two independent samples, we were able to exclude at least some external factors such as age-related developments (as the two samples differed in their age composition) or seasonality (Study 1 was conducted in Winter 2018, Study 2 in Summer 2021).

Assuming that our correlated changes were (mainly) caused by feedback loops between these constructs, future research should uncover how these feedback loops might work. We reviewed theories that assume that changes in the perception of major life events lead to changes in SWB as well as theories that assume the opposite direction of causality. In our exploratory analyses, we tried to gain first insights into the temporal order of the correlated changes. However, these analyses did not allow a definite conclusion. Thus, it remains a task

560 for future research to examine whether changes in the perception of major life events lead to 561 changes in SWB or vice versa. For example, longitudinal studies comprising multiple measurement occasions might be useful to better understand possible feedback mechanisms 
and the temporal order of the associations between the perception of major life events and SWB.

\section{Implications for Future Research on Major Life Events}

Although questions regarding the causality of the coupled changes between SWB and perceived event characteristics still need to be addressed, our findings nonetheless have practical implications for research on major life events. First, our study provides further evidence that examining how people perceive major life events may be useful to better understand their consequences (Luhmann et al., 2021). Second, to examine event-related changes, longitudinal study designs are necessary not only to address changes in the outcome of interest (Luhmann et al., 2014) but also to gain a more complete understanding of the perception of major life events and its changes over time. Third, as indicated by our robustness checks, the timing of the measurement occasions and the time passed since the event occurrence are relevant for the associations that can be found. For positive and negative affect, the first months after event occurrence seem to be particularly crucial.

\section{Limitations and Future Directions}

The two studies have several limitations. First, both studies relied on samples which were predominantly female and recruited in a Western democratic country. Thus, future research should examine whether our findings generalize to other cultural backgrounds. As the frequency of certain major life events differs among cultures, different associations between changes in SWB and changes in the perceived event characteristics might emerge in these populations (Ngo \& Le, 2007). Relatedly, in our studies, we only assessed the perceived event characteristics of the most important major life event the participants had experienced in the given time frame. Consequently, it remains a question whether other associations emerge when focusing on specific and maybe also less important events.

Second, as indicated above, assessing correlated changes between the perception of major life events and SWB is only a first step to learn more about their interplay. We found that changes in these constructs are related to each other. To amplify these findings, longitudinal studies with multiple measurement occasions and experimental studies (e.g., by manipulating people's SWB through interventions) should be conducted to learn more about the mechanisms causing the observed pattern of correlated changes.

Third, some associations between the perceived event characteristics and SWB emerged only in one of the two studies. In our robustness checks, we tried to examine whether differences in the designs of the two studies were responsible for differences in the results. However, these robustness checks can only be seen as an approximation to this question as not all possible reasons for differences could be examined. For example, we tried to estimate the influence of the Covid-19 pandemic in Study 2 by including a measure of Covid-related stress to the bivariate latent change score models, but the pandemic has consequences that 
600 reach far beyond people's self-rated stress (e.g., health problems, occurrence of different 601 major life events).

602 Fourth, in this article, we could not analyze which factors may have caused changes in 603 the perception of major life events as we observed naturally occurring changes in the perceived 604 event characteristics. However, for applied contexts like interventions, future research could 605 uncover under which conditions the perception of major life event changes. For example, 606 changes in the consequences of an event and changes in one's evaluation standard for major 607 life events may be possible candidates that elicit changes in the perception of major life events. 608

\section{Conclusion}

609 The present article further empathizes that considering the perception of major life 610 events enhances our understanding of event-related changes in SWB (Haehner, Kritzler et al., 611 2021; Luhmann et al., 2021). In particular, not only the initial perception of major life events, 612 but also their changes over time can be related to changes in SWB. Based on these findings, 613 future research should uncover the mechanisms causing this pattern of correlated changes. 


\section{References}

Abramson, L. Y., Metalsky, G. I., \& Alloy, L. B. (1989). Hopelessness depression: A theorybased subtype of depression. Psychological Review, 96(2), 358-372. https://doi.org/10.1037/0033-295X.96.2.358

Abramson, L. Y., Seligman, M. E., \& Teasdale, J. D. (1978). Learned helplessness in humans: Critique and reformulation. Journal of Abnormal Psychology, 87(1), 49-74. https://doi.org/10.1037/0021-843X.87.1.49

Allemand, M., \& Martin, M. (2016). On correlated change in personality. European Psychologist, 21(4), 237-253. https://doi.org/10.1027/1016-9040/a000256

Beck, A. T., \& Bredemeier, K. (2016). A unified model of depression. Clinical Psychological Science, 4(4), 596-619. https://doi.org/10.1177/2167702616628523

Brose, A., Blanke, E. S., Schmiedek, F., Kramer, A. C., Schmidt, A., \& Neubauer, A. B. (2021). Change in mental health symptoms during the COVID-19 pandemic: The role of appraisals and daily life experiences. Journal of Personality, 89(3), 468-482. https://doi.org/10.1111/jopy.12592

Busseri, M. A. (2018). Examining the structure of subjective well-being through meta-analysis of the associations among positive affect, negative affect, and life satisfaction. Personality and Individual Differences, 122, 68-71. https://doi.org/10.1016/i.paid.2017.10.003

Denissen, J. J. A., Luhmann, M., Chung, J. M., \& Bleidorn, W. (2019). Transactions between life events and personality traits across the adult lifespan. Journal of Personality and Social Psychology, 116(4), 612-633. https://doi.org/10.1037/pspp0000196

Diener, E. (1984). Subjective well-being. Psychological Bulletin, 95(3), 542-575. https://doi.org/10.1037/0033-2909.95.3.542

Diener, E., Emmons, R. A., Larsen, R. J., \& Griffin, S. (1985). The satisfaction with life scale. Journal of Personality Assessment, 49(1), 71-75. https://doi.org/10.1207/s15327752jpa4901 13

Diener, E., Suh, E. M., Lucas, R. E., \& Smith, H. L. (1999). Subjective well-being: Three decades of progress. Psychological Bulletin, 125(2), 276-302. https://doi.org/10.1037/0033-2909.125.2.276

Diener, E., Wirtz, D., Tov, W., Kim-Prieto, C., Choi, D.-w., Oishi, S., \& Biswas-Diener, R. (2010). New well-being measures: Short scales to assess flourishing and positive and negative feelings. Social Indicators Research, 97(2), 143-156. https://doi.org/10.1007/s11205-009-9493-y

Fassbender, I., Haehner, P., Buecker, S., \& Luhmann, M. (2021). Perceived characteristics of life events-short-term changes in prosociality and empathy? European Journal of Personality. Advance online publication. https://doi.org/10.1177/08902070211031762 
Frederick, S., \& Loewenstein, G. (1999). Hedonic adaptation. In D. Kahneman, E. Diener, \& N. Schwarz (Eds.), Well-Being: Foundations of Hedonic Psychology (pp. 302-329). Russell Sage Foundation.

Funder, D. C., \& Ozer, D. J. (2019). Evaluating effect size in psychological research: Sense and nonsense. Advances in Methods and Practices in Psychological Science, 2(2), 156-168. https://doi.org/10.1177/2515245919847202

Glaesmer, H., Grande, G., Braehler, E., \& Roth, M. (2011). The German version of the satisfaction with life scale (SWLS). European Journal of Psychological Assessment, 27(2), 127-132. https://doi.org/10.1027/1015-5759/a000058

Gross, J. J. (2002). Emotion regulation: Affective, cognitive, and social consequences. Psychophysiology, 39(3), 281-291. https://doi.org/10.1017/S0048577201393198

Haehner, P., Kritzler, S., Fassbender, I., \& Luhmann, M. (2021). Stability and change of perceived characteristics of major life events. Journal of Personality and Social Psychology. Advance online publication. https://doi.org/10.1037/pspp0000394

Haehner, P., Rakhshani, A., Fassbender, I., Lucas, R. E., Donnellan, M. B., \& Luhmann, M. (2021). Perception of major life events and personality trait change. PsyArXiv.

Haehner, P., Würtz, F., Kritzler, S., Kunna, M., Woud, M., \& Luhmann, M. (2022). Perception of major life events and depression: A systematic review [Manuscript in preparation].

Holmes, T. H., \& Rahe, R. H. (1967). The social readjustment rating scale. Journal of Psychosomatic Research, 11(2), 213-218. https://doi.org/10.1016/00223999(67)90010-4

Hou, W. K., Ng, S. M., \& Wan, J. H. Y. (2015). Changes in positive affect and mindfulness predict changes in cortisol response and psychiatric symptoms: A latent change score modelling approach. Psychology \& Health, 30(5), 551-567. https://doi.org/10.1080/08870446.2014.990389

Jorgensen, T. D., Pornprasertmanit, S., Schoemann, A. M., \& Rosseel, Y. (2020). semTools: Useful tools for structural equation modeling. https://CRAN.Rproject.org/package $=$ semTools

Kettlewell, N., Morris, R. W., Ho, N., Cobb-Clark, D. A., Cripps, S., \& Glozier, N. (2020). The differential impact of major life events on cognitive and affective wellbeing. SSM Population Health, 10, 100533. https://doi.org/10.1016/i.ssmph.2019.100533

Klimstra, T. A., Bleidorn, W., Asendorpf, J. B., van Aken, M. A.G., \& Denissen, J. J.A. (2013). Correlated change of Big Five personality traits across the lifespan: A search for determinants. Journal of Research in Personality, 47(6), 768-777. https://doi.org/10.1016/i.jrp.2013.08.004

Kritzler, S., Rakhshani, A., Terwiel, S., Fassbender, I., Donnellan, M. B., Lucas, R. E., \& Luhmann, M. (2021). How are common major life events perceived? Exploring 
688

689

690

691

692

693

694

695

696

697

698

699

700

701

702

703

704

705

706

707

708

709

710

711

712

713

714

715

716

717

718

719

720

721

722

723

differences between and variability of different typical event profiles and raters. PsyArXiv. https://doi.org/10.31234/osf.io/fncz3

Lazarus, R. S., \& Folkman, S. (1984). Stress, appraisal, and coping. Springer. http://site.ebrary.com/lib/alltitles/docDetail.action?doclD=10265641

Little, T. D. (2013). Longitudinal structural equation modeling. Methodology in the social sciences. Guilford Press. http://gbv.eblib.com/patron/FullRecord.aspx?p=1137447

Lucas, R. E. (2007). Adaptation and the set-point model of subjective well-being. Current Directions in Psychological Science, 16(2), 75-79. https://doi.org/10.1111/j.14678721.2007.00479.x

Luhmann, M., Fassbender, I., Alcock, M., \& Haehner, P. (2021). A dimensional taxonomy of perceived characteristics of major life events. Journal of Personality and Social Psychology, 121(3), 633-668. https://doi.org/10.1037/pspp0000291

Luhmann, M., Hofmann, W., Eid, M., \& Lucas, R. E. (2012). Subjective well-being and adaptation to life events: A meta-analysis. Journal of Personality and Social Psychology, 102(3), 592-615. https://doi.org/10.1037/a0025948

Luhmann, M., \& Intelisano, S. (2018). Hedonic adaptation and the set point for subjective well-being. In E. Diener, S. Oishi, \& L. Tay (Eds.), Handbook of well-being. DEF Publishers.

Luhmann, M., Orth, U., Specht, J., Kandler, C., \& Lucas, R. E. (2014). Studying changes in life circumstances and personality: It's about time. European Journal of Personality, 28(3), 256-266. https://doi.org/10.1002/per.1951

Mayer, J. D., McCormick, L. J., \& Strong, S. E. (1995). Mood-Congruent Memory and Natural Mood: New Evidence. Personality and Social Psychology Bulletin, 21(7), 736-746. https://doi.org/10.1177/0146167295217008

McArdle, J. J. (2009). Latent variable modeling of differences and changes with longitudinal data. Annual Review of Psychology, 60, 577-605. https://doi.org/10.1146/annurev.psych.60.110707.163612

Ngo, H. M., \& Le, T. N. (2007). Stressful life events, culture, and violence. Journal of Immigrant and Minority Health, 9(2), 75-84. https://doi.org/10.1007/s10903-0069018-6

Oishi, S. (2006). The concept of life satisfaction across cultures: An IRT analysis. Journal of Research in Personality, 40(4), 411-423. https://doi.org/10.1016/i.jrp.2005.02.002

Olaru, G., \& Allemand, M. (2021). Correlated personality change across time and age. European Journal of Personality, 089020702110140. https://doi.org/10.1177/08902070211014054

Prizmić-Larsen, Z., Kaliterna-Lipovčan, L., Larsen, R., Brkljačić, T., \& Brajša-Žganec, A. (2020). The role of flourishing in relationship between positive and negative life 

events and affective well-being. Applied Research in Quality of Life, 15(5), 14131431. https://doi.org/10.1007/s11482-019-09743-y

Rahm, T., Heise, E., \& Schuldt, M. (2017). Measuring the frequency of emotions-validation of the Scale of Positive and Negative Experience (SPANE) in Germany. PloS One, 12(2), e0171288. https://doi.org/10.1371/journal.pone.0171288

Rosseel, Y. (2012). lavaan: An R Package for Structural Equation Modeling. Journal of Statistical Software, 48(2). https://doi.org/10.18637/jss.v048.i02

Sarason, I. G., Johnson, J. H., \& Siegel, J. M. (1978). Assessing the impact of life changes: Development of the Life Experiences Survey. Journal of Consulting and Clinical Psychology, 46(5), 932-946. https://doi.org/10.1037//0022-006x.46.5.932

Schermelleh-Engel, K., Moosbrugger, H., \& Müller, H. (2003). Evaluating the fit of structural equation models: Tests of significance and descriptive goodness-of-fit measures. Methods of Psychological Research, 8(2), 23-74.

Shigemoto, Y. (2020). Reciprocal influence between posttraumatic stress and posttraumatic growth approximately one year after Hurricane Harvey: A bivariate latent change score modeling approach. Traumatology, 26(3), 317-324. https://doi.org/10.1037/trm0000231

Slavich, G. M., \& Sacher, J. (2019). Stress, sex hormones, inflammation, and major depressive disorder: Extending Social Signal Transduction Theory of Depression to account for sex differences in mood disorders. Psychopharmacology, 236(10), 30633079. https://doi.org/10.1007/s00213-019-05326-9

Specht, J., Egloff, B., \& Schmukle, S. C. (2011). The benefits of believing in chance or fate. Social Psychological and Personality Science, 2(2), 132-137. https://doi.org/10.1177/1948550610384635

Vries, J. H. de, Spengler, M., Frintrup, A., \& Mussel, P. (2021). Personality development in emerging adulthood-How the perception of life events and mindset affect personality trait change. Frontiers in Psychology, 12, 671421. https://doi.org/10.3389/fpsyg.2021.671421

Wang, Y. A., \& Rhemtulla, M. (2021). Power analysis for parameter estimation in structural equation modeling: A discussion and tutorial. Advances in Methods and Practices in Psychological Science, 4(1), 251524592091825. https://doi.org/10.1177/2515245920918253

Wilson, T. D., \& Gilbert, D. T. (2008). Explaining away: A model of affective adaptation. Perspectives on Psychological Science, 3(5), 370-386. https://doi.org/10.1111/j.1745-6924.2008.00085.x 
760 Yap, S. C.Y., Anusic, I., \& Lucas, R. E. (2014). Does happiness change? Evidence from 761 longitudinal studies. In K. M. Sheldon \& R. E. Lucas (Eds.), Stability of Happiness 762 (pp. 127-145). Elsevier. https://doi.org/10.1016/B978-0-12-411478-4.00007-2 


\section{Figure 1}

764 Illustration of the Univariate and Bivariate Latent Change Score Models

A

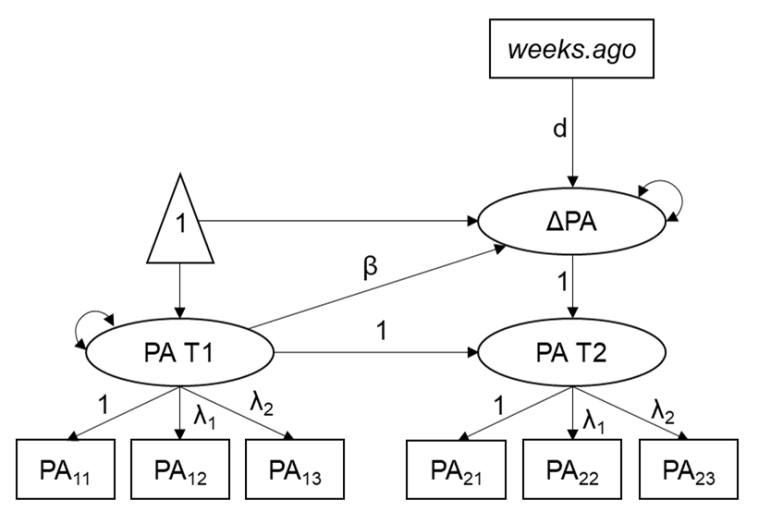

B

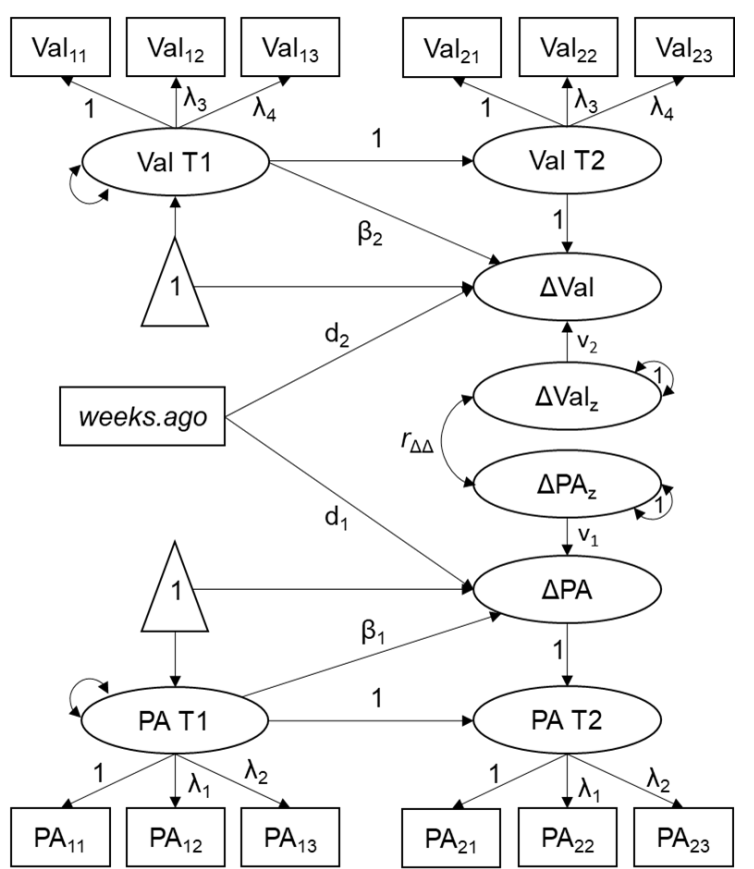

765 Note. Panel A: Illustration of the univariate latent change score models for positive affect. Positive affect at T1 (PA T1), positive affect at T2 (PA T2), and a latent change score

$766(\triangle \mathrm{PA})$ are the estimated latent variables in this model. The latent change score is controlled for the temporal distance since the event occurrence (weeks.ago). Intercepts of indicators, 767 residual variances, and residual covariances are not depicted. Panel B: Illustration of the bivariate latent change score models for positive affect and valence. In addition to the 768 latent change scores $(\Delta \mathrm{PA}, \Delta \mathrm{Val})$, this model includes two phantom variables $\left(\Delta \mathrm{PA}_{\mathrm{z}}, \Delta \mathrm{Val} \mathrm{z}_{\mathrm{z}}\right)$ with a variance fixed to 1 . These phantom variables allow estimating the correlation 769 between the latent change scores $(r \Delta \Delta)$ instead of their covariance. Intercepts of indicators, residual variances, and residual covariances are not depicted. PA = positive affect; Val $=$ valence 


\section{Table 1}

Descriptive Statistics for SWB and Perceived Event Characteristics for Study 1

\begin{tabular}{|c|c|c|c|c|c|c|c|c|c|c|c|c|}
\hline \multirow[t]{2}{*}{ Variables } & \multicolumn{4}{|c|}{ T1 } & \multicolumn{4}{|c|}{$\mathrm{T} 2$} & \multicolumn{4}{|c|}{ Latent Change Scores } \\
\hline & $N$ & $M$ & $S D$ & $\alpha$ & $N$ & $M$ & $S D$ & $\alpha$ & $N$ & $M$ & $S D$ & $M_{z}{ }^{\mathrm{a}}$ \\
\hline Life satisfaction & 619 & 5.11 & 1.21 & 0.83 & 433 & 5.18 & 1.22 & 0.84 & 619 & 0.18 & 0.49 & 0.37 \\
\hline Positive affect & 618 & 3.78 & 0.75 & 0.84 & 433 & 3.71 & 0.74 & 0.85 & 619 & -0.07 & 0.46 & -0.14 \\
\hline Negative affect & 618 & 2.59 & 0.84 & 0.78 & 433 & 2.62 & 0.8 & 0.75 & 619 & 0.01 & 0.50 & 0.03 \\
\hline Challenge & 618 & 3.42 & 1.07 & 0.84 & 433 & 3.47 & 0.97 & 0.82 & 619 & 0.02 & 0.44 & 0.04 \\
\hline Change in world views & 615 & 2.83 & 0.85 & 0.73 & 433 & 2.88 & 0.83 & 0.74 & 619 & 0.14 & 0.39 & 0.36 \\
\hline Emotional significance & 618 & 4.03 & 0.84 & 0.82 & 433 & 3.95 & 0.87 & 0.82 & 619 & -0.13 & 0.31 & -0.42 \\
\hline External control & 615 & 2.48 & 1.01 & 0.76 & 433 & 2.56 & 1.04 & 0.82 & 619 & 0.08 & 0.41 & 0.18 \\
\hline Extraordinariness & 617 & 2.33 & 0.96 & 0.77 & 433 & 2.33 & 0.94 & 0.76 & 619 & 0.01 & 0.52 & 0.03 \\
\hline Impact & 618 & 3.92 & 0.87 & 0.72 & 433 & 3.84 & 0.93 & 0.76 & 619 & -0.08 & 0.15 & -0.57 \\
\hline Predictability & 618 & 3.83 & 1.21 & 0.92 & 433 & 3.83 & 1.20 & 0.91 & 619 & 0.05 & 0.37 & 0.13 \\
\hline Social status change & 617 & 1.42 & 0.67 & 0.81 & 433 & 1.39 & 0.68 & 0.85 & 619 & -0.03 & 0.31 & -0.09 \\
\hline Valence & 618 & 3.86 & 1.27 & 0.95 & 433 & 3.90 & 1.20 & 0.95 & 619 & 0.03 & 0.34 & 0.09 \\
\hline
\end{tabular}

${ }^{\mathrm{a}} M_{z}=$ standardized mean of the latent change scores (calculated by dividing the mean of the latent change scores by their standard deviation). 


\section{Table 2}

Correlations Between Latent Change Score Factors in Study 1

\begin{tabular}{|c|c|c|c|c|c|c|c|c|c|c|c|c|}
\hline \multirow{2}{*}{$\begin{array}{l}\text { Perceived Event } \\
\text { Characteristics }\end{array}$} & \multicolumn{4}{|c|}{ Life satisfaction } & \multicolumn{4}{|c|}{ Positive affect } & \multicolumn{4}{|c|}{ Negative affect } \\
\hline & $r_{\Delta \Delta}$ & $z$ & $p$ & $99 \% \mathrm{CI}\left(r_{\Delta \Delta}\right)$ & $r_{\Delta \Delta}$ & $z$ & $p$ & $99 \% \mathrm{CI}\left(r_{\Delta \Delta}\right)$ & $r_{\Delta \Delta}$ & $z$ & $p$ & $99 \% \mathrm{CI}\left(r_{\Delta \Delta}\right)$ \\
\hline Challenge & -.14 & -1.78 & .075 & {$[-.34, .06]$} & -.26 & -3.78 & $<.001$ & {$[-.44,-.08]$} & .31 & 4.26 & $<.001$ & {$[.12, .49]$} \\
\hline Emotional significance & -.02 & -0.23 & .821 & {$[-.23, .19]$} & -.16 & -2.26 & .024 & {$[-.35, .02]$} & .06 & 0.76 & .449 & {$[-.14, .26]$} \\
\hline External control & -.25 & -3.34 & .001 & {$[-.45,-.06]$} & -.19 & -2.63 & .009 & {$[-.37,-.00]$} & .09 & 1.22 & .222 & {$[-.10, .29]$} \\
\hline Impact & .12 & 1.22 & .223 & {$[-.13, .37]$} & -.09 & -1.01 & .315 & {$[-.32, .14]$} & -.04 & -0.42 & .673 & {$[-.28, .20]$} \\
\hline Predictability & .08 & 0.97 & .334 & {$[-.13, .28]$} & -.13 & -1.77 & .077 & {$[-.31, .06]$} & .07 & 0.83 & .404 & {$[-.14, .27]$} \\
\hline Social status change & -.28 & -4.01 & $<.001$ & {$[-.47,-.10]$} & -.11 & -1.58 & .115 & {$[-.28, .07]$} & .08 & 1.18 & .236 & {$[-.10, .27]$} \\
\hline Valence & .34 & 5.08 & $<.001$ & {$[.17, .51]$} & .19 & 3.01 & .003 & {$[.03, .35]$} & -.23 & -3.50 & $<.001$ & {$[-.40,-.06]$} \\
\hline
\end{tabular}




\section{Table 3}

Differences in the Study Design Between Study 1 and Study 2

\begin{tabular}{lll}
\hline \multicolumn{1}{c}{ Design aspect } & \multicolumn{1}{c}{ Study 1} & Study 2 \\
\hline Sample & Addressed to young adults: & Addressed to the general public: \\
& $M_{\text {age }}=21.48$ years $(S D=4.05)$ & $M_{\text {age }}=34.18$ years $(S D=12.02)$ \\
Life event assessment & Open answer field & Life event checklist \\
Time frame of life events & Rated life events of the last 3 months & Rated life events of the last 12 months \\
Event Characteristics Questionnaire ${ }^{\text {a }}$ & 37-item version (as in Study 5 of Luhmann et al., & 38-item version (final version proposed by \\
& $2021)$ & Luhmann et al., 2021) \\
Scale of Positive and Negative Experiences & 6-item version (3 items per construct) & 12-item version (6 items per construct) \\
Satisfaction With Life Scale & 3-item version & 5-item version \\
Data collection period & 2018 to 2019 (before the Covid-19 pandemic) & 2021 (during the Covid-19 pandemic)
\end{tabular}

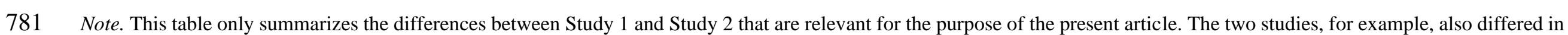

782 other constructs that were not used in the present article.

783 a For more details on the overlapping and non-overlapping items of the two versions of the Event Characteristics Questionnaire see Table S10. 


\section{Table 4}

Descriptive Statistics for SWB and Perceived Event Characteristics for Study 1

\begin{tabular}{|c|c|c|c|c|c|c|c|c|c|c|c|c|}
\hline \multirow[t]{2}{*}{ Variables } & \multicolumn{4}{|c|}{ T1 } & \multicolumn{4}{|c|}{$\mathrm{T} 2$} & \multicolumn{4}{|c|}{ Latent Change Scores } \\
\hline & $N$ & $M$ & $S D$ & $\alpha$ & $N$ & $M$ & $S D$ & $\alpha$ & $N$ & $M$ & $S D$ & $M_{z}{ }^{\mathrm{a}}$ \\
\hline Life satisfaction & 691 & 4.59 & 1.34 & 0.9 & 438 & 4.56 & 1.41 & 0.92 & 691 & -0.05 & 0.42 & -0.11 \\
\hline Positive affect & 689 & 3.23 & 0.87 & 0.94 & 438 & 3.32 & 0.88 & 0.94 & 691 & 0.10 & 0.49 & 0.20 \\
\hline Negative affect & 688 & 2.70 & 0.86 & 0.87 & 438 & 2.54 & 0.89 & 0.89 & 691 & -0.15 & 0.34 & -0.44 \\
\hline Challenge & 689 & 3.28 & 1.27 & 0.9 & 437 & 3.23 & 1.20 & 0.89 & 691 & -0.04 & 0.34 & -0.13 \\
\hline Change in world views & 688 & 3.10 & 1.12 & 0.9 & 438 & 3.12 & 1.10 & 0.91 & 691 & 0.01 & 0.52 & 0.01 \\
\hline Emotional significance & 691 & 3.94 & 0.95 & 0.83 & 437 & 3.75 & 1.01 & 0.85 & 691 & -0.13 & 0.39 & -0.33 \\
\hline External control & 690 & 2.33 & 1.21 & 0.88 & 438 & 2.41 & 1.19 & 0.89 & 691 & 0.06 & 0.53 & 0.12 \\
\hline Extraordinariness & 687 & 2.49 & 0.86 & 0.63 & 438 & 2.53 & 0.90 & 0.72 & 691 & 0.02 & 0.33 & 0.05 \\
\hline Impact & 688 & 3.55 & 1.12 & 0.85 & 438 & 3.48 & 1.13 & 0.85 & 691 & -0.11 & 0.31 & -0.34 \\
\hline Predictability & 688 & 3.19 & 1.38 & 0.92 & 438 & 3.2 & 1.36 & 0.92 & 691 & 0.03 & 0.53 & 0.07 \\
\hline Social status change & 689 & 1.48 & 0.82 & 0.88 & 438 & 1.58 & 0.92 & 0.91 & 691 & 0.08 & 0.34 & 0.23 \\
\hline Valence & 689 & 3.34 & 1.57 & 0.97 & 438 & 3.35 & 1.49 & 0.96 & 691 & 0.04 & 0.42 & 0.09 \\
\hline
\end{tabular}

${ }^{\mathrm{a}} M_{z}=$ standardized mean of the latent change scores (calculated by dividing the mean of the latent change scores by their standard deviation). 


\section{Table 5}

Correlations Between Latent Change Scores Factors in Study 2

\begin{tabular}{|c|c|c|c|c|c|c|c|c|c|c|c|c|}
\hline \multirow{2}{*}{$\begin{array}{l}\text { Perceived Event } \\
\text { Characteristics }\end{array}$} & \multicolumn{4}{|c|}{ Life satisfaction } & \multicolumn{4}{|c|}{ Positive affect } & \multicolumn{4}{|c|}{ Negative affect } \\
\hline & $r_{\Delta \Delta}$ & $z$ & $p$ & $95 \% \mathrm{CI}\left(r_{\Delta \Delta}\right)$ & $r_{\Delta \Delta}$ & $z$ & $p$ & $95 \% \mathrm{CI}\left(r_{\Delta \Delta}\right)$ & $r_{\Delta \Delta}$ & $z$ & $p$ & $95 \% \mathrm{CI}\left(r_{\Delta \Delta}\right)$ \\
\hline Challenge & -.11 & -1.49 & .136 & {$[-.25, .03]$} & -.19 & -2.98 & .003 & {$[-.31,-.06]$} & .19 & 2.72 & .006 & {$[.05, .32]$} \\
\hline Change in world views & .03 & 0.43 & .668 & {$[-.10, .16]$} & -.03 & -0.44 & .659 & {$[-.14, .09]$} & .05 & 0.84 & .400 & {$[-.07, .18]$} \\
\hline Emotional significance & .00 & 0.02 & .981 & {$[-.14, .14]$} & -.09 & -1.43 & .154 & {$[-.22, .03]$} & .05 & 0.73 & .467 & {$[-.08, .18]$} \\
\hline External control & -.18 & -2.64 & .008 & {$[-.32,-.05]$} & -.02 & -0.39 & .697 & {$[-.14, .10]$} & .03 & 0.41 & .682 & {$[-.10, .16]$} \\
\hline Extraordinariness & .05 & 0.62 & .536 & {$[-.10, .20]$} & .03 & 0.42 & .672 & {$[-.10, .16]$} & -.09 & -1.20 & .232 & {$[-.23, .06]$} \\
\hline Impact & -.02 & -0.19 & .845 & {$[-.17, .14]$} & -.15 & -2.24 & .025 & {$[-.28,-.02]$} & .08 & 1.16 & .246 & {$[-.06, .23]$} \\
\hline Predictability & -.01 & -0.18 & .854 & {$[-.15, .12]$} & -.03 & -0.53 & .598 & {$[-.15, .09]$} & -.02 & -0.29 & .772 & {$[-.15, .11]$} \\
\hline Social status change & -.17 & -2.47 & .014 & {$[-.31,-.04]$} & -.14 & -2.22 & .026 & {$[-.26,-.02]$} & .17 & 2.60 & .009 & {$[.04, .30]$} \\
\hline Valence & .04 & 0.54 & .592 & {$[-.09, .17]$} & .10 & 1.79 & .074 & {$[-.01, .22]$} & -.06 & -1.03 & .301 & {$[-.19, .06]$} \\
\hline
\end{tabular}




\section{Table 6}

Overview on the Results of the Robustness Checks

\begin{tabular}{|c|c|c|}
\hline Factor that was tested & How it was tested & Results \\
\hline $\begin{array}{l}\text { Different items used in the } \\
\text { two studies }\end{array}$ & $\begin{array}{l}\text { Estimated the bivariate latent change } \\
\text { score models only using the items which } \\
\text { were employed in both studies }\end{array}$ & $\begin{array}{ll}\text { - } & \text { Pattern of results remains similar (e.g., similar effect sizes; Table S21) } \\
\text { - } & \text { However, some correlations that were significant in the main analyses fell below our level of } \\
& \text { significance; mainly because standard errors were larger when only using overlapping items }\end{array}$ \\
\hline $\begin{array}{l}\text { Potential relevance of stress } \\
\text { due to the Covid-19 } \\
\text { pandemic }\end{array}$ & $\begin{array}{l}\text { Included Covid-19 related stress at } \mathrm{T} 1 \text { as } \\
\text { predictor of the latent change scores to } \\
\text { control for its effects }\end{array}$ & $\begin{array}{l}\text { Associations between latent change scores were almost identical compared to the main analyses } \\
\text { (Table S22) } \\
\text { Covid-19 related stress was negatively related to changes in valence }(p=.009) \text { and positively } \\
\text { related to changes in change in world views }(p=.003)\end{array}$ \\
\hline $\begin{array}{l}\text { Different target age groups of } \\
\text { the two studies }\end{array}$ & $\begin{array}{l}\text { Included age as predictors of the latent } \\
\text { change scores to control for its effects }\end{array}$ & $\begin{array}{l}\text { - Associations between latent change scores were almost identical compared to the main analyses } \\
\text { (Table S23) } \\
\text { - Age was not associated with changes in the perceived event characteristics and changes in the } \\
\text { SWB components (all } p \mathrm{~s}>.010 \text { ) }\end{array}$ \\
\hline $\begin{array}{l}\text { Different event categories } \\
\text { examined in the two studies }\end{array}$ & $\begin{array}{l}\text { Estimated the bivariate latent change } \\
\text { score models only using events that } \\
\text { were examined in both studies } \\
(N=533)\end{array}$ & $\begin{array}{l}\text { - Compared to the main analyses, associations were stronger using only the overlapping events } \\
\text { (Table S24) } \\
\text { - Changes in valence were significantly associated with changes in positive affect } \\
\text { - } \quad \text { As in Study 1, changes in social status change were not significantly associated with changes in } \\
\text { negative affect } \\
\text { - } \quad \text { In general: Results of Study } 2 \text { came more similar to the results of Study } 1\end{array}$ \\
\hline $\begin{array}{l}\text { Different time frames of the } \\
\text { included events in the two } \\
\text { studies }\end{array}$ & $\begin{array}{l}\text { Estimated the bivariate latent change } \\
\text { score models only using events that } \\
\text { occurred in the last three months } \\
(N=136)\end{array}$ & $\begin{array}{l}\text { - Compared to the main analyses, associations were stronger when only including events that } \\
\text { occurred in the last } 3 \text { months before T1 (Table S25) } \\
\text { - } \quad \text { However, due to the smaller sample size, most associations were not significant } \\
\text { - } \\
\text { Additional exploratory analyses indicated that associations between changes in the perceived } \\
\text { event characteristics and changes in positive and negative affect become weaker when less recent } \\
\text { are considered (Figure S1) }\end{array}$ \\
\hline
\end{tabular}

